\title{
Nível de informação a respeito de medicamentos prescritos a pacientes ambulatoriais de hospital universitário
}

\author{
Patient knowledge about drugs prescribed \\ in a teaching hospital
}

Tatiane da Silva 1

Eloir Paulo Schenkel 1

Sotero Serrate Mengue 2

\footnotetext{
1 Faculdade de Farmácia, Universidade Federal do Rio Grande do Sul. Av. Ipiranga 2752, Porto Alegre, RS 90610-000, Brasil. 2 Departamento de Medicina Social, Faculdade de Medicina, Universidade Federal do Rio Grande do Sul. Rua Ramiro Barcelos 2600 , sala 414, Porto Alegre, RS 90035-003, Brasil.
}

\begin{abstract}
Lack of information on medication is one of the main reasons why some 30-50\% of patients fail to take their medicines as prescribed. To assess patient knowledge about prescribed drugs, outpatients from an internal medicine clinic in a teaching hospital were interviewed after medical consultation. Patients were asked to identify the name, indication, dosage, administration schedule, duration of treatment, side effects, and precautions. Patients' answers were compared to medical prescription or patients' medical records. Two hundred and sixty-four patients were interviewed, of whom 34\% displayed satisfactory knowledge. In $31 \%$ of cases the drug name stated was incorrect, while in 19\% the reported indication differed from that on the medical records. Dosage and administration schedules stated by patients disagreed with physician's instructions in $19 \%$ and $31 \%$ of cases, respectively. The results suggest that the majority of outpatients have sufficient knowledge for the safe use of prescribed drugs, under an ideal outpatient setting.
\end{abstract}

Key words Drugs; Drug Prescription; Compliance; Drug Information

Resumo A ausência de informações acerca dos medicamentos constitui uma das principais razões pelas quais $30 \%$ a $50 \%$ dos pacientes não os usam conforme a prescrição. Com o objetivo de aferir o conhecimento relativo aos medicamentos prescritos, pacientes atendidos no ambulatório de Medicina Interna de um hospital universitário foram entrevistados após a consulta médica. O nível de informação foi verificado mediante perguntas relativas ao nome do medicamento, indicação, dose, freqüência de uso, duração do tratamento, efeitos adversos e precauções. As respostas foram comparadas com a receita médica e com o prontuário do paciente. Do total de 264 pacientes entrevistados, 34\% obtiveram bom nível de conhecimento. Em 31\% dos casos, o nome do medicamento foi declarado incorretamente e, em 19\%, a indicação de uso declarada pelo paciente discordou da indicação médica. No que se refere à dose e freqüência de administração, $19 \%$ e $31 \%$ das respostas, respectivamente, discordaram da prescrição médica. Os resultados sugerem que a maioria dos pacientes possui suficiente nível de informação para a utilização segura dos medicamentos prescritos sob condições ambulatoriais ideais.

Palavras-chave Medicamentos; Prescrição de Medicamentos; Cumprimento; Informação sobre Medicamentos 


\section{Introdução}

A não-adesão ao tratamento medicamentoso pode ser uma das razões pelas quais medicamentos reconhecidamente eficazes sob condições controladas resultam inefetivos quando utilizados na prática clínica habitual. Estudos realizados em diversos países indicam que 50\% a $60 \%$ dos pacientes que recebem uma prescrição não cumprem o tratamento estabelecido pelo médico, e muitos o interrompem quando se sentem melhores (Eraker et al., 1984; Becker, 1985; Nyazema et al., 1991).

A falta de informações a respeito da doença e do tratamento ou a não compreensão das informações recebidas dos profissionais da saúde podem ser determinantes para a não-adesão involuntária do paciente ao tratamento (Roizblatt et al., 1984). Segundo alguns autores, a falta de informações relativas ao medicamento é um dos principais fatores responsáveis pelo uso em desacordo com a prescrição médica por $30 \%$ a $50 \%$ dos pacientes (Kessler, 1991; Farley, 1995; Marwick, 1997).

Apesar da informação acerca dos medicamentos constituir condição básica para a adesão ao tratamento, não se tem prestado atenção suficiente ao seu fornecimento nas diversas situações de atendimento ao paciente, incluindo a consulta médica e a dispensação do medicamento em farmácias. Diante disso, diversas organizações internacionais, tais como a World Health Organization, Health Action International e a Food and Drug Administration, vêm alertando para a necessidade crescente de melhorar os aspectos educacionais relacionados com o paciente e os medicamentos prescritos. Nos Estados Unidos, um dos objetivos de um programa nacional para melhorar a saúde dos americanos - denominado Healthy People 2000 - é alcançar, até o final do século, um percentual de $75 \%$ de pacientes que tenham recebido informações escritas a respeito dos medicamentos vendidos sob prescrição médica (Marwick, 1995).

Os estudos que abordam o tema têm enfocado aspectos educacionais, econômicos, psicológicos, sociais e interpessoais relacionados ao cumprimento da prescrição. Pesquisa realizada no Brasil por Bakirtzief (1996) demonstrou que a adesão de pacientes em tratamento da hanseníase estava associada à satisfação com o atendimento do profissional médico. Os aspectos relativos especificamente à informação que o paciente possui acerca dos medicamentos prescritos têm sido, no entanto, pouco investigados no Brasil. Estudo feito por Azevedo (1987) a respeito do nível de informação concernente à digoxina - apresentado por pacientes hospitalizados em Salvador, Bahia - verificou que $27 \%$ dos pacientes apresentaram conhecimento insuficiente no que se refere ao medicamento, $50 \%$ regular e $11 \%$ bom. Nenhum estudo que enfocasse a informação quanto a medicamentos utilizados em nível ambulatorial realizado no Brasil foi localizado.

Nesse sentido, este trabalho foi desenvolvido com o objetivo de medir o nível de informação que pacientes atendidos no serviço ambulatorial de Medicina Interna de um hospital universitário apresentavam quanto aos medicamentos prescritos na última consulta médica.

\section{Método}

Local: o estudo foi realizado no ambulatório do Serviço de Medicina Interna do Hospital de Clínicas de Porto Alegre (HCPA). Este hospital universitário de nível terciário possui 725 leitos, integra o Sistema Único de Saúde e presta assistência em 61 especialidades e subespecialidades médicas. No ano de 1997, foram efetuadas 586.161 consultas, das quais cerca de 12.000, no Serviço de Medicina Interna.

Pacientes: foram incluídos pacientes maiores de 18 anos que se apresentaram à consulta médica desacompanhados, receberam indicação de uso de um ou mais medicamentos na consulta feita no dia da entrevista e estavam em condições de comunicar-se - isto é, pacientes não debilitados -, selecionados por meio de amostragem não-probabilística consecutiva entre o período de 29 de dezembro de 1997 e 15 de junho de 1998.

Coleta dos dados: os pacientes foram entrevistados por um dos autores na saída do ambulatório por intermédio de questionário previamente testado em estudo piloto. Aos entrevistados foi perguntado nome, indicação, dose, freqüência de administração, duração do tratamento, possíveis efeitos adversos e precauções quanto ao primeiro medicamento listado na receita médica. Quando o paciente não possuía receita, considerava-se o primeiro medicamento citado pelo paciente ao ser questionado a respeito dos medicamentos que tinham sido indicados na consulta recém feita. Durante a entrevista, permitia-se ao paciente consultar a receita ou qualquer outro material (embalagem do medicamento, anotações em papel etc.) para responder as questões relativas ao medicamento. $\mathrm{O}$ paciente era entrevistado somente após a sua permissão, mediante termo de consentimento assinado. Além das perguntas relacionadas ao nível de informação no que 
concernia ao medicamento, foram feitas perguntas respeitantes ao uso prévio do medicamento e às características sociodemográficas dos pacientes. Este estudo foi aprovado pelas Comissões Científica e de Pesquisa e Ética em Saúde do HCPA.

Análise dos dados: ao final da entrevista a receita médica foi transcrita e o prontuário foi posteriormente revisado para obter informações respeitantes ao medicamento prescrito e à indicação terapêutica. Na receita foram pesquisados os dados a respeito de nome, dose, freqüência de administração e duração do tratamento. Dados relativos à indicação terapêutica foram buscados primariamente no prontuário. Na ausência ou insuficiência dos dados da receita quanto aos itens da informação acima especificados, os registros médicos da consulta em questão foram examinados em busca dessas informações. A análise das respostas com referência a efeitos adversos e precauções foi baseada nas informações das monografias dos medicamentos da United States Pharmacopoea Drug Information (USP DI) (United States Pharmacopoea Drug Information, 1997).

A interpretação da existência de concordância entre as respostas dos pacientes e as informações presentes na receita e no prontuário foi feita independentemente por dois revisores. As respostas foram consideradas corretas quando era possível inferir que havia acordo entre as declarações dos pacientes e as instruções da receita e o prontuário, mesmo quando utilizadas terminologias diferentes. Foi exigida a concordância dos dois revisores para julgar correta a resposta do paciente. Em caso de discordância, um terceiro revisor foi consultado. Foram excluídas das análises individuais - quanto à concordância entre as respostas dos pacientes e os itens relacionados com as informações do medicamento - as entrevistas em que não foi possível verificar a existência ou não de concordância com base apenas nas informações apresentadas na receita ou no prontuário.

Além da análise individual dos itens da informação investigados, foi desenvolvido um escore, pelo qual foi atribuído diferentes pontos para cada item, de acordo com a sua importância para a utilização segura dos medicamentos em nível ambulatorial. Partindo da definição de uso seguro do medicamento como utilização que não cause danos à saúde e ao bem-estar do paciente, foram definidos três níveis de informação: a) nível bom, o qual dá condições para o paciente usar o medicamento de forma segura em qualquer circunstância; b) nível regular, o qual dá condições para o pa- ciente usar o medicamento de forma segura em condições ideais sem nenhum tipo de intercorrência durante o tratamento; c) nível insuficiente, o qual não dá condições para o paciente utilizar o medicamento com segurança. Dessa forma, foram atribuídos dois pontos para nome do medicamento, dose e freqüência de administração e um ponto para duração do tratamento, indicação terapêutica, efeitos adversos e precauções, totalizando o máximo de dez pontos. Pacientes que somaram menos de seis pontos foram classificados como apresentando nível de informação insuficiente; aqueles com seis a oito pontos foram classificados como tendo nível de informação regular; e os com mais de oito pontos foram classificados como tendo nível de informação bom.

A classificação do nível de informação em "insuficiente", "regular" e "bom” aqui proposta serve como indicativo para avaliar se os pacientes estão em condições de utilizar os medicamentos prescritos com segurança. Considerando que os aspectos incluídos possuem diferentes graus de importância para a utilização segura do medicamento em nível ambulatorial, foram atribuídos pesos diferentes a cada item. Para os itens imprescindíveis à correta aquisição e administração do medicamento foi atribuído peso dois. Para os outros itens que não costumam influenciar decisivamente na administração correta do medicamento, mas que, dependendo da ocorrência de eventos inesperados, podem ganhar maior importância, foi atribuído peso um.

\section{Resultados}

De um total de 277 pacientes elegíveis, 11 não aceitaram participar do estudo. No todo, foram 266 os entrevistados, dentre os quais dois foram posteriormente excluídos da análise por falta de informações suficientes encontradas na receita ou prontuário.

Como mostra a Tabela 1, os pacientes incluídos neste estudo foram predominantemente do sexo feminino (75\%), adultos (idade média de 57 anos) e com primeiro grau incompleto de escolaridade (em média, 5 anos de escolaridade). Do total de pacientes entrevistados, $78 \%$ já haviam utilizado uma vez, ao menos, o medicamento analisado. Foram indicados 2,4 medicamentos por paciente, em média. Os medicamentos mais freqüentes na amostra foram propranolol (14\%), verapamil (7\%), hidroclorotiazida (7\%), captopril $(4 \%)$, nifedipina (4\%), paracetamol (4\%), enalapril (3\%), femprocumona (3\%) e levotiroxina (3\%). 
Tabela 1

\begin{tabular}{|c|c|}
\hline Características & Valores \\
\hline \multicolumn{2}{|l|}{ Sexo (n) } \\
\hline Feminino & $198(75 \%)$ \\
\hline Masculino & $66(25 \%)$ \\
\hline Idade (em anos) & $57 \pm 13$ \\
\hline Escolaridade (em anos) & $4,9 \pm 3,6$ \\
\hline Renda familiar (em reais) & $662 \pm 698$ \\
\hline \multicolumn{2}{|l|}{ Classe social } \\
\hline Burguesia & $5(2 \%)$ \\
\hline Nova pequena burguesia & $10(4 \%)$ \\
\hline Pequena burguesia tradicional & $60(23 \%)$ \\
\hline Proletariado não-típico & $65(25 \%)$ \\
\hline Proletariado típico & $81(31 \%)$ \\
\hline Subproletariado & $37(14 \%)$ \\
\hline \multicolumn{2}{|l|}{ Receita } \\
\hline Recebeu & 195 (74\%) \\
\hline Não recebeu & $69(26 \%)$ \\
\hline Número de medicamentos indicados & $2,4 \pm 1,4$ \\
\hline Número de consultas no último ano & $3,4 \pm 1,9$ \\
\hline \multicolumn{2}{|l|}{ Tempo de utilização do serviço } \\
\hline Mais de 1 ano & $162(63 \%)$ \\
\hline Menos de 1 ano & $87(34 \%)$ \\
\hline 1 ano & $8(3 \%)$ \\
\hline \multicolumn{2}{|l|}{ Uso prévio do medicamento } \\
\hline Sim & $206(78 \%)$ \\
\hline Não & $55(21 \%)$ \\
\hline Não sabe & $3(1 \%)$ \\
\hline $\begin{array}{l}\text { Tempo de uso prévio } \\
\text { do medicamento (em meses) }\end{array}$ & $40 \pm 53$ \\
\hline
\end{tabular}

A Tabela 2 sumariza a concordância entre as respostas dos pacientes e as informações presentes na receita e prontuário médico para os itens analisados.

Nome do medicamento. O índice de acerto para o item nome do medicamento foi de $69 \%$. Foram consideradas respostas corretas todas aquelas em que a resposta do paciente não permitia confundir o medicamento prescrito com o nome de outro medicamento qualquer. Respostas dadas em nome comercial para medicamento prescrito pelo nome genérico e vice-versa foram aceitas.

Indicação terapêutica. Um dos itens em que se verificou maior freqüência na concordância entre a resposta do paciente e a informação presente na receita ou no prontuário refere-se à indicação terapêutica (81\%). As respostas relativas à indicação foram avaliadas como corretas, desde que correspondessem ao principal órgão do aparelho ou sistema do organismo em que o medicamento atua ou onde se situa o sintoma tratado, independente das diferenças na terminologia utilizada.

Dose. Em $81 \%$ das respostas quanto ao item dose verificou-se concordância entre a resposta do paciente e a informação obtida na receita ou prontuário. As respostas foram julgadas corretas quando se referiam à mesma quantidade em unidades de medida (mg., g., ml) ou de forma farmacêutica (comprimidos, cápsulas) a ser administrada em cada horário ou turno, estabelecida pelo médico, confirmada na receita médica ou, na ausência desta, no prontuário médico.

Freqüência de administração. Em relação à freqüência de administração do medicamento, $69 \%$ das respostas apresentaram concordância com a receita e/ou prontuário. Foram aceitas respostas dadas em número de vezes por dia ou intervalo entre doses, conforme tenha sido escrito na receita. Pacientes que responderam em intervalo entre doses (por exemplo, de oito em oito horas) quando na receita constava vezes por dia (por exemplo, três vezes por dia) tiveram suas respostas aceitas como corretas. $\mathrm{O}$ mesmo não aconteceu com o oposto, ou seja, quando eram especificados horários ou intervalos determinados entre doses (de oito em oito horas) não eram aceitas respostas do tipo três vezes por dia à pergunta "de quantas em quantas horas deve tomar o remédio?"

Duração do tratamento. Quanto à duração do tratamento, foi verificada uma concordância de $61 \%$ nas respostas dos pacientes à pergunta "por quanto tempo o Sr. (Sra.) deve tomar esse remédio?" Para tratamentos agudos, com tempo de duração bem definido, como, por exemplo, tratamentos com antimicrobianos, as respostas dos pacientes foram julgadas com base na receita médica ou prontuário. Para os tratamentos crônicos, responsáveis pela maior parte dos casos analisados, foram consideradas corretas as respostas "até a próxima consulta" e "por toda a vida", indicando que o medicamento deveria ser tomado enquanto a avaliação do médico julgasse adequado o prosseguimento do tratamento.

Precauções. Um dos itens em que foi comprovada a menor freqüência de acerto na resposta do paciente diz respeito às respostas à pergunta "Existem cuidados especiais que o Sr. (Sra.) deve ter em relação ao uso deste remédio?". De 264 entrevistados, 36\% responderam 
Concordância entre a resposta do paciente e a indicação médica em relação ao nome do medicamento, indicação terapêutica, dose a ser administrada em cada horário, freqüência de administração e duração do tratamento.

\begin{tabular}{llll}
\hline Informação & n* & Freqüência & $\%$ \\
\hline Nome do medicamento & 257 & 178 & 69 \\
Indicação terapêutica & 243 & 197 & 81 \\
Dose & 240 & 194 & 81 \\
Freqüência de administração & 232 & 161 & 69 \\
Duração do tratamento & 264 & 161 & 61 \\
\hline
\end{tabular}

* o tamanho da amostra final é diferente para cada item analisado devido às exclusões mencionadas no texto.

"sim". A análise das monografias incluídas na USP DI revelou que apenas $19 \%$ das respostas tinham alguma relação com as precauções relativas ao medicamento em questão (United States Pharmacopoea Drug Information, 1997).

Efeitos adversos. Do total de 264 entrevistados, somente $20 \%$ responderam "sim" à pergunta "Esse remédio pode causar reações desagradáveis?”. Comparando com as informações disponíveis nas monografias da USP DI quanto aos possíveis efeitos adversos do medicamento, foi confirmado que somente $16 \%$ correspondiam a efeitos adversos freqüentes e/ou graves.

De acordo com o escore proposto para a análise do nível de informação concernente aos medicamentos prescritos, foi possível agrupar os pacientes em três níveis de informação (Tabela 3). Da mesma forma que para a análise individual dos itens da informação, foram excluídos dessa análise os pacientes para os quais não foi possível avaliar a concordância de uma ou mais respostas referentes aos itens propostos. Do total de 199 pacientes, 34\% apresentaram bom nível de informação a respeito do medicamento prescrito, $57 \%$ regular e $9 \%$ insuficiente.

\section{Discussão}

A presente investigação foi desenvolvida com o propósito de verificar o nível de informação de pacientes ambulatoriais no que diz respeito à indicação terapêutica, ao nome, à dose, à freqüência de administração, à duração do tratamento, aos efeitos adversos e às precauções, itens importantes para a aquisição e utilização segura do medicamento.

Tomando em conta que os laboratórios farmacêuticos podem alterar a embalagem, forma e cor de medicamentos já disponíveis no
Tabela 3

Nível de informação básica sobre os medicamentos prescritos, de acordo com a importância de cada item para a sua utilização adequada $(n=199)$.

\begin{tabular}{lcc}
\hline Nível de informação básica & Freqüência & $\%$ \\
\hline $\begin{array}{l}\text { Insuficiente } \\
\text { (menos de 6 pontos) }\end{array}$ & 18 & 9 \\
$\begin{array}{l}\text { Regular } \\
\text { (6 a 8 pontos) }\end{array}$ & 115 & 57 \\
$\begin{array}{l}\text { Bom } \\
\text { (mais de 8 pontos) }\end{array}$ & 68 & 34 \\
\hline
\end{tabular}

comércio sob determinada apresentação, e que o paciente pode mudar de especialidade farmacêutica conforme a disponibilidade no mercado e o preço, julgou-se ser o nome do medicamento sua melhor forma de identificação para o paciente. Tendo-se em mente que a prática de prescrição pelo nome genérico ainda não se tornou corrente no meio médico, incluindo o serviço no qual o trabalho foi realizado, não foi restringida a identificação apenas ao nome genérico, aceitando-se qualquer nome comercial que identificasse corretamente o medicamento prescrito. O percentual de acerto para esse item foi superior ao encontrado em estudo similar feito por Olivares \& Espinoza (1996) - no caso, 46\% -, mas apresentou nível inferior ao de outro estudo que envolvia pacientes ambulatoriais psiquiátricos, realizado por Tempier (1996), e que alcançou $93 \%$.

Mesmo com a possibilidade de consultar a receita médica para responder às questões relativas à dose e à freqüência de administração (número de vezes por dia, intervalo entre doses ou horário específico do dia, para os casos de administração diária única), cerca de $20 \%$ e 
$30 \%$ dos pacientes, respectivamente, demonstraram não saber a dose e a freqüência de administração. Somado ao fato de que a maioria desses pacientes já estava utilizando os medicamentos, o percentual de pacientes que não sabia quando e quanto devem usar é clinicamente expressivo, tendo em vista que esses itens são imprescindíveis ao cumprimento do esquema terapêutico estabelecido pelo médico.

Uma pequena parcela de entrevistados forneceu resposta quanto à duração do tratamento em dias, semanas ou meses, correspondendo aos casos para os quais foram indicados tratamentos com duração definida, como na antibioticoterapia de infecções respiratórias e urinárias. Tendo em vista que a amostra é composta predominantemente por pacientes portadores de alguma doença crônica - em especial, hipertensão arterial sistêmica, diabete e hipotireoidismo -, a elevada parcela de pacientes que forneceu respostas do tipo "indefinido", "sempre", "por toda a vida" ou "pelo menos até a próxima consulta" condiz com as características da amostra incluída no estudo.

A insuficiência de informações com respeito aos efeitos adversos observada pode estar relacionada à valoração secundária dada a esse item da informação, tanto por quem fornece a prescrição e o medicamento, quanto por quem o utiliza. A possibilidade de o medicamento causar efeitos adversos acaba sendo, muitas vezes, omitida pelo prescritor, receoso de que informações negativas relativas ao medicamento prejudiquem a adesão do paciente ao tratamento ou mesmo que, por auto-sugestão, o paciente venha a sentir o efeito adverso comentado.

As informações apresentadas pelos pacientes no que se refere a precauções durante o uso do medicamento prescrito, observadas nas respostas do tipo "manter longe do alcance de crianças", "tomar as doses corretas" e "tomar nos horários", correspondem a cuidados com a freqüência de administração ou com o armazenamento.

A aferição do conhecimento do paciente a respeito do medicamento prescrito tem sido feita de modos distintos nos estudos que abordam o tema. Alguns pesquisadores têm analisado os diversos itens do conhecimento de forma individual (Fletcher et al., 1979; Olivares \& Espinoza, 1996). Outros, por sua vez, têm usado escores ou pontuações que sumarizam os itens analisados (Azevedo, 1987; Vargas et al., 1991). Outros, ainda, utilizam um conceito global de conhecimento que envolve a compreensão da doença e do tratamento (Nyazema et al., 1991). Conforme demonstrado em estudo de- senvolvido por Ascione et al. (1986), o conceito "conhecimento sobre o medicamento" inclui diferentes itens que não são suficientemente equivalentes na representação do conceito. Nesse sentido, é preciso distinguir a importância de cada item analisado de acordo com as características do paciente, do tratamento medicamentoso e das condições em que o medicamento será utilizado.

Em conformidade com os pesos atribuídos aos diferentes aspectos avaliados, os resultados da classificação utilizada demonstram que por volta de um terço dos pacientes entrevistados possui nível de informação bom, suficiente para a utilização segura do medicamento em condições ambulatoriais. A maior parte dos pacientes apresenta nível de informação regular, ou seja, suficiente para a administração adequada do medicamento em condições ideais, sem considerar, porém, a possibilidade de ocorrência de eventos, durante o curso do tratamento, que possam pôr em risco a sua efetividade, tais como o esquecimento de doses, o aparecimento de reações adversas inesperadas, o uso concomitante de outros medicamentos sem conhecimento do médico, e a dificuldade de adequar os horários de administração do medicamento à rotina de atividades do paciente, entre outras.

Finalmente, os resultados do escore proposto apontam para a existência de pequena parcela de pacientes que não apresenta conhecimento mínimo para a administração segura do medicamento em qualquer circunstância. Embora constitua um grupo pequeno de pacientes, sua existência é clinicamente relevante, já que a relação risco/benefício do uso do medicamento é potencialmente desfavorável para esses pacientes, independente do diagnóstico e escolha terapêutica corretamente formulados.

\section{Conclusões}

Diante dos resultados obtidos, conclui-se que a maioria dos pacientes atendidos no ambulatório do Serviço de Medicina Interna do HCPA apresenta nível de informação suficiente para a administração do medicamento de acordo com a prescrição médica, sob condições ambulatoriais ideais.

Em uma época na qual, em qualquer situação e em qualquer lugar, a informação constitui bem de valor inestimável e ferramenta de sobrevivência nas relações profissionais, comerciais e pessoais, não se admite que os seres humanos, estando doentes e, por isso mesmo, 
fragilizados, ao necessitarem consumir um produto medicamentoso qualquer, venham a ingerir comprimidos sem saber para que servem, como usá-los e que cuidados adotar para minimizar ou prevenir potenciais riscos a sua saúde. Em uma época na qual os direitos dos consumidores estão explicitados na legislação da maioria dos países; em que a terapêutica medicamentosa combate doenças que, até há algumas décadas atrás eram incuráveis, a informação a respeito do medicamento é essen- cial. Nesse sentido, é preciso que sejam aprimorados os meios de fornecer a informação ao paciente. Isso envolve a formação dos profissionais responsáveis pela educação relativa aos medicamentos, a reorganização do tempo dedicado à orientação quanto à prescrição no consultório, a atuação direta do farmacêutico na dispensação dos medicamentos, bem como a conscientização dos profissionais e dos pacientes no que concerne ao valor da informação.

\section{Referências}

ASCIONE, F. J.; KIRSCHT, J. P. \& SHIMP, L. A., 1986. An assessment of different components of patient medication knowledge. Medical Care, 24:10181028.

AZEVEDO, N. D. A., 1987. Conhecimento do paciente hospitalizado sobre o uso de digital. Revista Baiana de Enfermagem, 3:95-111.

BAKIRTZIEF, Z., 1996. Identificando barreiras para aderência ao tratamento de hanseníase. Cadernos de Saúde Pública, 12:497-505.

BECKER, M. H., 1985. Patient adherence to prescribed therapies. Medical Care, 23:539-555.

ERAKER, S. A.; KIRSCHT, J. P. \& BECKER, M. H., 1984. Understanding and improving patient compliance. Annals of Internal Medicine, 100:258-268.

FARLEY, D., 1995. FDA proposes program to give patients better medication information. FDA Consumer Magazine, 29. 26 April 1997. <http://www. fda.gov/fdac/features/995_medinfo.html>

FLETCHER, S. W.; FLETCHER, R. H.; THOMAS, D. C. \& HAMANN, C., 1979. Patients' understanding of prescribed drugs. Journal of Community Health, 4:183-189.

KESSLER, D. A., 1991. Communicating with patients about their medications. New England Journal of Medicine, 325:1650-1652.

MARWICK, C., 1995. FDA pushes for prescription drug information. JAMA, 273:1815-1816.
MARWICK, C., 1997. MedGuide: At last a long-sought opportunity for patient education about prescription drugs. JAMA, 277:949-950.

NYAZEMA, N. Z.; CHAVUNDUKA, D.; DZIMWASHA, M.; MADONDO, F.; MAFANA, E. \& MBEWE, A., 1991. Drug information for the community: Type and source. Central African Journal of Medicine, 37:203-206.

OLIVARES, J. \& ESPINOZA, Y., 1996. Determinación del nivel de información sobre el uso de antiinflamatorios no esteroideos en pacientes ambulatorios de un hospital general. Medicamentos y Salud Popular, 33:19-28.

ROIZBLATT, A. S.; CUETO, G. \& ALVAREZ, P., 1984. Diagnóstico y tratamiento. Que saben los pacientes? Boletín de la Oficina Sanitaria Panamericana, 97:491-495.

TEMPIER, R., 1996. Long-term psychiatric patients' knowledge about their medication. Psychiatric Services, 47:1385-1387.

UNITED STATES PHARMACOPOEA DRUG INFORMATION, 1997. Drug Information for the Health Care Professional. Massachussetts: Rand MacNally.

VARGAS, M.; DOMECQ, C. \& MAUREIRA, C., 1991. Análisis del grado de cumplimiento y de conocimiento del tratamiento entre los pacientes ingresados en un Servicio de Medicina. Revista de la O.F.I.L., 1:2-10. 